\title{
Challenges in Using Seismocardiography for Blood Pressure Monitoring
}

\author{
Kasper Soerensen ${ }^{1}$, Ajay K Verma ${ }^{2}$, Andrew Blaber ${ }^{3}$, John Zanetti ${ }^{2}$, Samuel Emil Schmidt ${ }^{1}$, Johannes \\ $\mathrm{J} \mathrm{Struijk}^{1}$, Kouhyar Tavakolian ${ }^{2}$ \\ ${ }^{1}$ Aalborg University - Department of Health Science and Technology, Aalborg, Denmark \\ ${ }^{2}$ University of North Dakota, Grand Forks, USA \\ ${ }^{3}$ Simon Fraser University, Vancouver, Canada
}

\begin{abstract}
The capability of continuously and non-invasive blood pressure (BP) monitoring is valuable in the clinical setting. Pulse transit time (PTT) has shown the promise to track changes in the arterial BP. This study examines the efficacy of seismocardiogram (SCG) and photoplethysmogram (PPG) combination for timing the proximal and distal pulse respectively of a pulse wave. The PTT is defined as a difference between the proximal and distal timing of a pulse wave. Methods: A total of 18 subjects participated in the study. Subjects were subjected to a lower-body negative pressure (LBNP) protocol to -60 mmHg. Electrocardiogram (ECG), SCG, PPG and BP was recorded simultaneously during the LNBP protocol. Subjects that endured negative pressure to $-40 \mathrm{mmHg}$, had $S C G$ signals with distinct $A O$ points and for whom the average $B P$ was lower in the final stage of $L B N P$ compared to supine baseline were included in data analysis. A simple logarithmic model was used to estimate BP based on PTT. Estimated BP was then correlated with PTT. Results: A total of 7 subjects were included in the final data analysis. On an average the subjects systolic BP dropped during the LBNP protocol and PTT was shortened. None of these changes were significant. For 3 subjects the correlation between PTT and estimated systolic blood pressure was significant. Conclusion: The current study showed a trend toward shorter PTT as blood pressure lowers, but the trend was weak and not consistent.
\end{abstract}

\section{Introduction}

Seismocardiography (SCG) is currently being revisited as a simple non-invasive technique for measuring cardiac contraction, after the technology was largely abandoned in the late 80 's $[1,2]$. SCG is a measure of the vibration created by the contraction of the heart, usually measured on the xiphoid process with an accelerometer. The SCG morphology has distinctive fiducial points making it possible to detect aortic-valve opening (AO) among other, see Fig 1 [2].

Pulse transit time (PTT) is the time it takes a pulse wave to travel between proximal and distal arterial sites. Typically, the R-peak of the electrocardiogram (ECG) is used for timing the proximal pulse while the foot of the pulse-photoplethysmogram (PPG) is a marker of the distal pulse. The morphology of the ECG challenges the accuracy of the timing of AO [3]. The PTT obtained using the ECG-PPG combination has especially been challenged in the literature as a surrogate for blood pressure (BP) due to the inclusion of the isovolumic contraction period [4]. In contrast the $\mathrm{SCG}$ morphology has a distinct $\mathrm{AO}$ point, thus, in this research we explore the potential of SCG AO as a marker of the proximal timing of PTT.

PTT is correlated with BP [4]. Monitoring PTT can therefore be used for obtaining continuous and noninvasive estimates of BP. Changes in the BP are monitored physiologically by baroreceptors. Momentary fall in BP will cause an increase in peripheral vascular resistance and cardiac output to keep the BP at a sufficient level, which would result in a shortening in PTT. However, if the body is not capable of upholder the BP (as it would be the case in haemorrhage) the BP would fall resulting in a prolonged PTT.

Using the SCG AO as proximal timing for PTT has been done before [3]. That study was limited though by the BP maneuverer protocol and suggests a study with subjects placed in a lower body negative pressure (LBNP) chamber [3] to gain greater control over the BP.

In this study we investigate the potential of using SCG $\mathrm{AO}$ and PPG onset on to track near beat-by-beat changes during a LBNP protocol.

\section{Method}




\subsection{Data Collection and Experimental Design}

Data was collected from a total of 18 healthy subjects. (age: $29 \pm 6$ years, height: $171 \pm 8 \mathrm{~cm}$, weight: $69 \pm 12 \mathrm{~kg}$ ). For all subjects simultaneous recordings of ECG, SCG on the Xiphoid Process and PPG from the finger was obtained.

PPG was recorded continuously using photoplethysmography cuff placed on the mid-phalanx of the left hand middle finger (FMS, Amsterdam, The Netherlands), the ECG was acquired in a lead II configuration using Lifepak8 system (Medtronic Inc, MN, USA). All signals were acquired using NI 9205 analog input module (National Instruments Inc, TX, USA) at a sampling rate of $1000 \mathrm{~Hz}$. The beat-to-beat systolic and diastolic blood pressure values were determined using Beatscope software (Finapres FMS, The Netherlands).

Data acquisition was performed in the Aerospace Physiology lab in the Department of Biomedical Physiology and Kinesiology. The experimental protocol was approved by the research ethics board of Simon Fraser University as a minimum risk. All participants signed the informed consent form prior to any experimentation.

Subjects lay supine in the LBNP chamber for 5 minutes for baseline recording. After which the chamber pressure was gradually reduced to $-20 \mathrm{mmHg}$, after this point on the chamber pressure was reduced in a steps of $10 \mathrm{mmHg}$ until $-60 \mathrm{mmHg}$ or the onset of presyncope. Five minutes of simultaneous data were acquired during each LBNP stage. The experimentation was terminated immediately if the participant requested and a registered nurse was present for the safety of participants while the experimentation was in progress.

\subsection{Signal processing}

Because of the low pressure in the LBNP chamber the subjects may experience pain, causing them to move on the bed during the experiment. This movement can distort the SCG signal due to the high sensitivity of the accelerometer. Furthermore, not all subjects went through all the stages of negative pressure, due to experience of pain with the low pressure. For the data analysis subjects with visually distinct SCG AO and high quality PPG signals were included. The subject was only included if recordings from rest to $-40 \mathrm{mmHg}$ LBNP was obtained.

For each stage of LNBP the last 120 seconds of data (ECG, SCG, PPG and blood pressure) was extracted. The SCG and PPG signals were segmented into beats based on detection of R-peaks. This segmentation was performed as described in [5].

\subsubsection{Fiducial Point Detection}

A semi-automatic algorithm was developed to detect the AO point in the SCG signals. This algorithm is inspired by the algorithm described in [6].

For each beat the Shannon-energy was computed for the first heart sound (S1) using a high pass filtered version of the SCG signal as a proxy for sound. Based on this envelope a window was extracted starting 80 milliseconds before the peak of the envelope. In this window the SCG signal was band pass filtered from $0.01 \mathrm{~Hz}$ to $90 \mathrm{~Hz}$. The mitral valve closure (MC) point was detected in this window as either a peak or the shoulder to the steepest descent.

Next, the isovolumic moment (IM) point was detected as the lowest point in a window spanning from the MC point to the peak envelope point found in the first step. A backtracking algorithm was then used to ensure that the correct MC point was found, based on the newly found IM point. This algorithm detects the first peak or shoulder prior to the IM point as the MC point. In some cases this point is not in the same location as the MC point found at first.

Lastly the AO point was detected as the highest peak following the IM point.

\subsubsection{PPG Onset Detection}

In both[3] and [4] the tangent intersection method is used with the best results for detection of PPG onset, why the same technique is used in this study. For each beat the tangent intersection was calculated and used as the PPG onset point.

\subsection{Data Analysis}

For all subjects the data was visualized before segmentation to ensure high quality of the SCG signal with a distinct $\mathrm{AO}$ point.

After applying the segmentation, fiducial point detection and PPG onset detection all signals were manually verified to ensure that the correct locations were found in all signals (see example in Fig 1). If any of the algorithms had failed the algorithm was adjusted ensuring the right points were detected. 

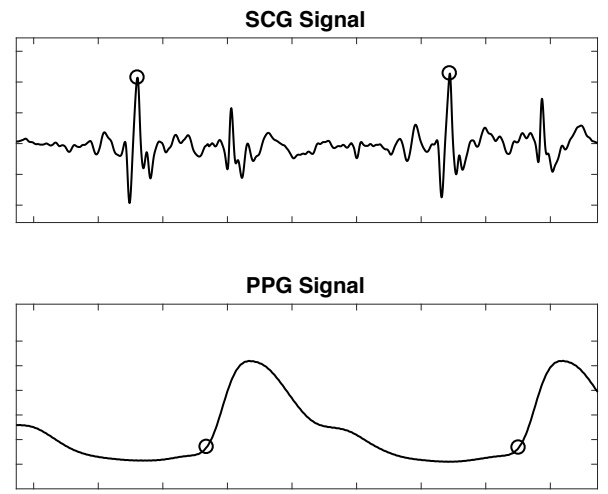

Fig 1 - Top: SCG signal with AO point markings. Buttom: PPG signal with onset markings. Both signals are typical examples of SCG and PPG optained during the LBNP protocol.

The transit time from the AO point to the onset of the PPG pulse wave was calculated for all the beats. Based on the last 30 beats in each pressure level an average value of the PTT and blood pressure of 5 beats were calculated, resulting in 6 samples for each pressure level. These were concatenated with samples for each of the four levels resulting in 24 samples in total for each subject.

Systolic (BPS), diastolic (BPD) and mean arterial (MAP) BP were modeled with a simple logarithmic model as in $[3,4]$. This model is based on the Moens-Korteweg [7] equation and elasticity model by Hughes [8]:

$$
B P=K_{1} \cdot \ln (P T T)+K_{2}(1)
$$

The model (1) is used to estimate the BP values based on the PTT, using two subject and case specific parameters $\left(\mathrm{K}_{1}\right.$ and $\left.\mathrm{K}_{2}\right)$. The parameters were estimated for each of the three BPs for each subject. The subject specific parameters were found using a least squares method. The results from the estimation of BP were also correlated with PTT.

Furthermore, for each subject average BPs and PTT was calculated for each level of LBNP including rest. The average BPS, BPD, MAP and PTT for all subjects was used in an ANOVA to determine if changes to any of the parameters were significant. A P-value $<0.05$ was considered significant.

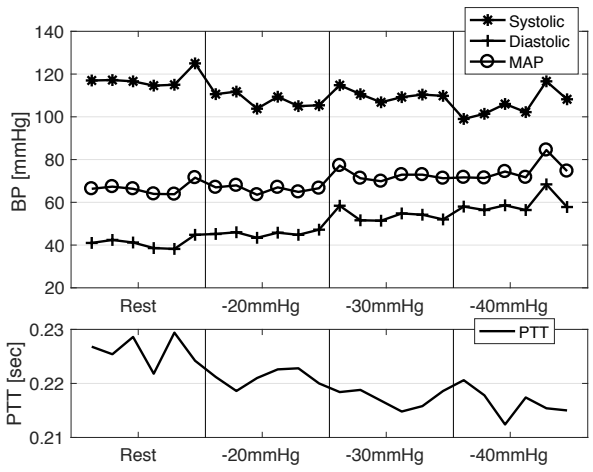

Fig 2- BP and PPT plotted over time in the four stages in the LBNP protocol.

\section{$3 \quad$ Results}

11 subjects had recordings from rest, $-20,-30$ and $40 \mathrm{mmHg}$. Signals from these subjects had a distinct AO point and continuous PPG recording. One subject was removed due to the Finapres device not functioning correctly. For the remaining 10 subjects, those for which the BPS was higher at $-40 \mathrm{mmHg}$ LBNP compared to rest were excluded. This left 7 subjects for data analysis.

Table 1 shows the average blood pressure and PTT for all remaining subjects during rest and the three levels of negative pressure.

Despite a trend toward lower SBP and PTT the differences were not significant.

Table 2 shows the correlation for each subject between PTT and the three BPs estimated with the model (1). Pvalues below 0.05 are considered significant and marked with an asterisk $(*)$.

Table 1. Average BP and PTT for all subjects across the three LBNP levels and rest.

\begin{tabular}{lllll}
\hline & $\begin{array}{l}\text { BPS } \\
{[\mathrm{mmHg}]}\end{array}$ & $\begin{array}{l}\text { BPD } \\
{[\mathrm{mmHg}]}\end{array}$ & $\begin{array}{l}\text { MAP } \\
{[\mathrm{mmHg}]}\end{array}$ & $\begin{array}{l}\text { PTT } \\
{[\mathrm{msec}]}\end{array}$ \\
\hline Rest & $125 \pm 15$ & $63 \pm 18$ & $84 \pm 16$ & $237 \pm 18$ \\
$-20 \mathrm{mmHg}$ & $119 \pm 20$ & $63 \pm 19$ & $82 \pm 18$ & $236 \pm 19$ \\
$-30 \mathrm{mmHg}$ & $117 \pm 16$ & $66 \pm 17$ & $84 \pm 16$ & $234 \pm 22$ \\
$-40 \mathrm{mmHg}$ & $112 \pm 17$ & $69 \pm 17$ & $83 \pm 16$ & $232 \pm 20$ \\
\hline
\end{tabular}

Table 2. Correlation between PTT and BP estimated using the model (1).

\begin{tabular}{llll}
\hline Subject no. & Systolic & Diastolic & MAP \\
\hline 1 & $0.45^{*}$ & $0.81^{*}$ & $0.68^{*}$ \\
2 & $0.43^{*}$ & 0.36 & 0.03 \\
3 & $-0.48^{*}$ & 0.06 & 0.33 \\
4 & 0.40 & $0.47^{*}$ & 0.04 \\
5 & 0.35 & 0.31 & 0.07 \\
6 & 0.37 & 0.37 & 0.14 \\
7 & 0.06 & $0.43^{*}$ & 0.31 \\
\hline
\end{tabular}

\section{Discussion}

This study examines the potential for using SCG and PPG to estimate blood pressure during a LBNP protocol. Even though we see changes in blood pressure in some subjects, during the negative pressure levels, these changes are only weakly reflected in the PTT, see Table 2.

The correlation between the blood pressure and PTT was low and inconsistently one subject demonstrated an negative correlation between SBP and PTT.

That only $70 \%$ of the included subjects expired a drop in systolic blood pressure and that PTT did not strictly follow the systole blood pressure shows that the 
cardiovascular response to the LBNP chamber is not fully predictable.

There are some limitations to this work including the very low number of useable subjects. While we see slight decline in systolic blood pressure at $-40 \mathrm{mmHg}$ LBNP, going to -50 or $-60 \mathrm{mmHg}$ might have introduced a significantly lower blood pressure in the subjects and possibly also a prolonged PTT. The BP device was not always correctly calibrated between sessions of measurement. On this basis the exact blood pressure values should not be trusted, although we consider the measured changes in blood pressure to be accurate.

The lower BP should result in a lower tension in the arterial wall which would normally mean a higher PTT, as observed by Payne et al [9]. However, in this experiment there will be a reduced venous return to the heart from the legs, which decreases stroke volume. The baroreceptors in the aortic arch will compensate this decline in stroke volume by increasing the heart rate and potentially by vasoconstriction. Higher heart rate will typically decrease the pulse pressure (difference between BPS and BPD) which is also seen in fig 2 and the vasoconstriction will lead to lower PTT as we observed in most cases.

This means that PTT relates to BP, but the direction of these changes is not always specific.

In conclusion the current study showed a trend toward lower PTT as blood pressure lowers, but the trend was weak and not consistent. This points toward that the cardiovascular response to the LBNP chamber is complex and that a fall in blood pressure does not automatically lead to a prolonged PTT.

\section{References}

[1] Giovangrandi L, Inan OT, Wiard RM, et al. Ballistocardiography - A method worth revisiting. Proc Annu Int Conf IEEE Eng Med Biol Soc EMBS 2011; 4279-4282.

[2] Inan O, Migeotte P-F, Park K-S, et al. Ballistocardiography and Seismocardiography: A Review of Recent Advances. IEEE J Biomed Heal Informatics 2014; PP: 1-1.

[3] Yang C, Tavassolian N. Pulse Transit Time Measurement Using Seismocardiogram, Photoplethysmogram, and Acoustic Recordings: Evaluation and Comparison. IEEE J Biomed Heal Informatics 2017; 2194: 1-1.

[4] Kim CS, Carek AM, Mukkamala R, et al. Ballistocardiogram as proximal timing reference for pulse transit time measurement: Potential for cuffless blood pressure monitoring. IEEE Trans Biomed Eng 2015; 62: 2657-2664.

[5] Jensen AS, Schmidt SE, Struijk JJ, et al. Effects of Cardiac Resynchronization Therapy on the First Heart Sound Energy. 2014; 29-32.

[6] Khosrow-Khavar F, Tavakolian K, Blaber A, et al. Automatic Annotation of Seismocardiogram with High Frequency Precordial Accelerations. IEEE J Biomed Heal informatics 2014; 2194: 1428-1434.

[7] Milnor WR. Hemodynamics.

[8] Hughes DJ, Babbs CF, Geddes LA, et al. Measurements of Young's modulus of elasticity of the canine aorta with ultrasound. Ultrason Imaging 1979; 1: 356-367.

[9] Payne RA, Symeonides CN, Webb DJ, et al. Pulse transit time measured from the ECG: an unreliable marker of beat-to-beat blood pressure. J Appl Physiol 2006; 100: 136-141.

Address for correspondence.

Kasper Soerensen.

Fredrik Bajers Vej C2-203

9220 Aalborg

ks@hst.aau.dk 